\title{
Towards Multi-DOF Model Mediated Teleoperation: Using Vision to Augment Feedback
}

\author{
Bert Willaert*, Jeannette Bohg ${ }^{\dagger}$, Hendrik Van Brussel* and Günter Niemeyer ${ }^{\ddagger}$ \\ ${ }^{*}$ Dept. of Mechanical Engineering, KU Leuven, Belgium \\ ${ }^{\dagger}$ Max Planck Institute for Intelligent Systems, Tübingen, Germany \\ ${ }^{\ddagger}$ Willow Garage Inc., Menlo Park, USA \\ Email: gunter.niemeyer@stanford.edu
}

\begin{abstract}
In this paper, we address some of the challenges that arise as model-mediated teleoperation is applied to systems with multiple degrees of freedom and multiple sensors. Specifically we use a system with position, force, and vision sensors to explore an environment geometry in two degrees of freedom. The inclusion of vision is proposed to alleviate the difficulties of estimating an increasing number of environment properties. Vision can furthermore increase the predictive nature of model-mediated teleoperation, by effectively predicting touch feedback before the slave is even in contact with the environment.

We focus on the case of estimating the location and orientation of a local surface patch at the contact point between the slave and the environment. We describe the various information sources with their respective limitations and create a combined model estimator as part of a multi-d.o.f. model-mediated controller. An experiment demonstrates the feasibility and benefits of utilizing vision sensors in teleoperation.
\end{abstract}

Index Terms-Teleoperation - Model-mediation - Multi-modal estimation

\section{INTRODUCTION}

Bilateral teleoperation systems allow the user to act on a remote environment via a robotic slave device while providing force feedback through a master device. The realization of bilateral teleoperation is most challenging in the presence of an inevitable amount of (phase or time) lag between the master and slave. Lags can be due to limited response times of local controllers, or due to substantial communication time-delays. In both cases, classical control approaches typically result in either poor transparency or poor stability properties [1], [2].

These scenarios form a great potential for the modelmediated approach [3], [4], [5], which counteracts the loop lag by the use of lead compensation: the models predict the response of the environment or human operator. In most previous works on model-mediated teleoperation, the model structure was chosen to be consistent with the environment. Hence, for an unchanging environment, the model estimator converges to a constant and stability is achieved by assumption even in the presence of a large loop lag.

The value of teleoperation is, however, highest for operations in unstructured environments which are at best difficult to model. So models should be interpreted as time-varying signals without a correct or constant steady state value [6], [7]. This observation reintroduces the stability question but also largely extends the usability of the model-mediated approach.
However, in order to realize the full potential of modelmediated teleoperation, more research is needed on how to extend the approach to multi-d.o.f. scenarios. So far, most model-mediated teleoperation research focused on single-d.o.f. scenarios and the environment model described a geometric contact property [4], [5], [8] or a dynamic contact property [9], [10], [11], [12], [13]. For multi-d.o.f. scenarios, the model estimation itself faces increased complications. Apart from the fact that geometric and dynamic properties often have to be estimated simultaneously, there is also an explosion of the number of measurements and model parameters that is involved:

- In case of a single-d.o.f. scenario, the dynamic properties of the environment can be represented with the impedance $Z_{e}$. In an n-d.o.f. scenario, however, the dynamics have to be represented by an $\mathrm{n} \times \mathrm{n}$ impedance matrix $\mathbf{Z}_{e}$, i.e. $\mathrm{n}^{2}$ instead of one transfer functions have to be defined.

- In the same way, the geometric description of an object in a single-d.o.f. scenario can be obtained after one motion. Finding the geometric description of a simple plane, however, already requires three motions: one to define the location and two to define the orientation of the plane.

Due to this explosion of the number of model parameters, simple position and force measurements can no longer be linked to the model parameters in a comprehensible way.

In the domain of autonomous robots, online contact and environment modelling is an active research topic [14], [15], [16]. In [16] e.g., the measured end-effector position, velocity and force are not only used to estimate the parameters of environmental contact dynamics but also geometric parameters such as environment position and orientation. Apart from the work described in [17], environment modelling for multid.o.f. scenarios received little or no attention in the domain of teleoperation. In [17], a 2-d.o.f. model-mediated teleoperation is described, modelling the environment geometrically as a set of rigid planes. The location and orientation of a plane are estimated through a heuristic approach using force and position sensor measurements.

The use of vision sensors can contribute to both the model estimation challenge as well as to the predictive nature of model-mediation:

- Vision sensors can be used to obtain the full geometric properties of the environment from a single image. As 
such, they alleviate the model estimation challenge for multi-d.o.f. scenarios.

- In [6], [7], it was explained how model-mediated teleoperation counteracts the lag between the master and the slave by using lead compensation: the models predict the response of the environment and/or human operator. Vision sensors can bring the predictive nature of modelmediation to a next level by allowing model estimation before physical contact takes places.

However, vision-based sensing also has limitations. First, the sensor data can be noisy or incomplete due to poor visibility conditions or due to occlusion by the manipulator interacting with the environment you are trying to observe. In other words, there is no guarantee of getting data. Second, vision can not observe force-based information. Dynamic properties, such as friction, impedance, texture, etc. are typically only detectable by touch and direct manipulation. Hence, vision has a complementary nature to touch-based sensing.

In this paper, we leverage the respective advantages of vision and force-based sensing and begin to integrate the sensing modalities for model-mediated feedback in a multid.o.f. scenario. To do so, we focus on the simple case of planar 2-d.o.f. operations.

Section $\Pi$ describes the considered scenario and the model. Section [II describes all information sources used to estimation the model-parameters, while the actual estimation strategy is defined in Section IV, The controller and the experiments are described in Section $\mathrm{V}$ and Section VI respectively. The paper ends with conclusions in Section VII

\section{THE PLANAR SCENARIO}

This paper describes a 2-d.o.f. model-mediated controller with a model-estimator that uses different sources of information for the scenario shown in Fig. 1(a) In past work [6], [7], it was claimed that the model should be treated as a time-varying signal: the model should predict locally and over a short period of time while allowing quick estimation and adapting to the system movements. Assuming the objects in the environment to be locally planar, we propose a simple yet powerful model of a flat rigid surface.

The model contains a surface location $\vec{p}$ and a surface normal $\vec{n}$ pointing into the surface. The top view in Fig. 1(a) shows these model parameters. Note that this model can be interpreted as a multi-d.o.f. extension of the object location model described in [5], [7]. A curved environment surface is modeled as a locally planar segment that has to change its orientation as the contact location moves. Obviously smaller radii of curvature need faster model changes and challenge the algorithm, as we will see below. For simplicity in this first study, we have considered neither compliant objects nor corners or edges which would require model jumps [7].

As shown in Fig. 1(b), the experimental setup consists of the left arm of the PR2 robot and a haptic device designed and built at the University of Leuven [18]. We confined both robot motions to the plane and linked the $\mathrm{x} / \mathrm{y}$ Cartesian axes. The gripper of the PR2 arm is holding a stick in order to

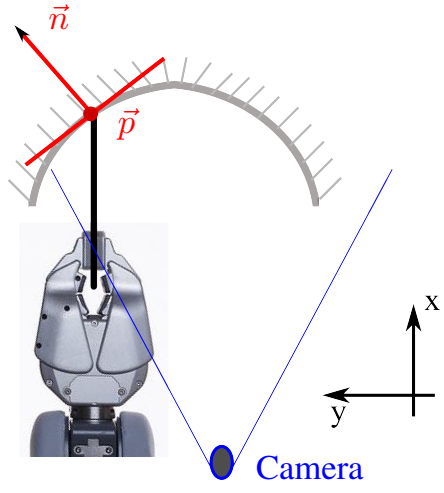

(a)

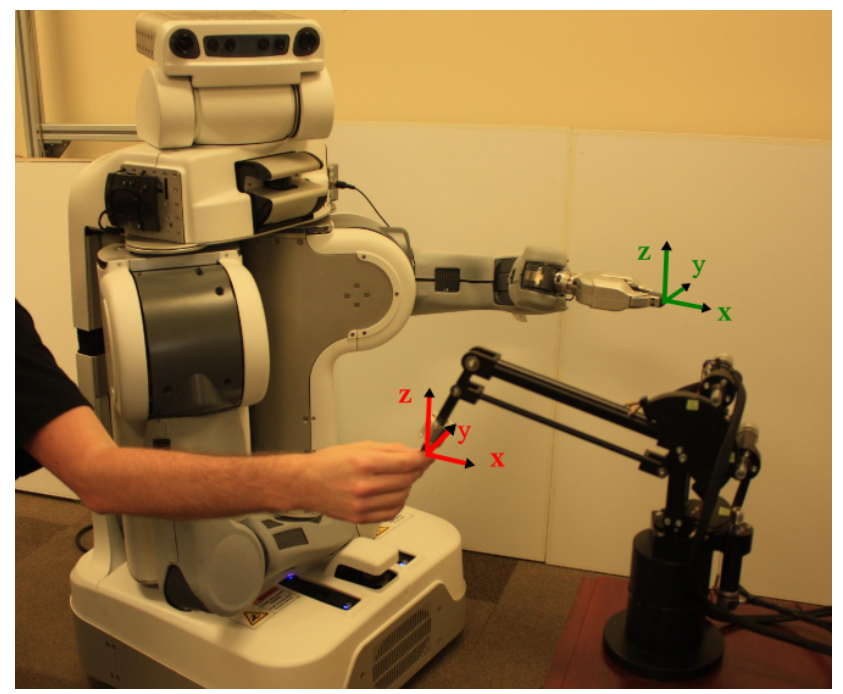

(b)

Fig. 1. (a) A top view of the considered scenario for multi-sensor based model estimation and (b) the experimental setup.

guarantee point contacts. The tip of this stick is considered the end-effector of the slave and referred to as $\vec{x}_{s}$.

\section{Model INFORMation SOURCES}

To estimate the model parameters $\vec{p}$ and $\vec{n}$, the following sources are available: the force sensor at the end-effector of the slave, the position measurements in the joints, a stereo camera in conjunction with a textured light projector observing the environment and also the estimation history. Each (combination of) source(s) exhibits its own precision and reliability.

\section{A. Force information: $\vec{n}_{F}$}

When applying force to an object, the orientation of the force vector $\vec{F}_{e}$ gives an estimate for the orientation of the object. However, due to the presence of friction in the contact, this estimated normal $\vec{n}_{F}$ can deviate up to $45^{\circ}$ from the actual normal of the object in the contact point, in case a maximum friction coefficient of one is assumed. Hence, this information should only be used as a last resort.

\section{B. Force and Position information: $\vec{p}_{P}$}

Each time the magnitude of the interaction force with the environment, $\left\|\vec{F}_{e}\right\|$, exceeds a pre-defined threshold $F_{\text {thres }}$, the 
slave is assumed to be in contact and the actual position of the end-effector gives an estimate for the location of the object: $\vec{p}_{P}$. Due to the flexibility and the backlash of the PR2-arm, there is an uncertainty of more than $10 \mathrm{~mm}$ on the position measurement for the end-effector.

\section{Force and Trajectory information: $\vec{n}_{T}$}

While moving along a rigid surface, the surface normal must be orthogonal to the direction of motion. Hence, the surface normal can be estimated based on the location of the current contact point and a set of previous contact points.

To this end, a 2-d.o.f. spatial filter is defined that remembers $\mathrm{n}_{b}$ contact points that are well spread over a specific spatial constant $d_{T}$. At each sample time for which the magnitude of the interaction force with the environment is higher than a predefined threshold $F_{\text {thres }}$, an old contact point is replaced by a new contact point in the buffer in such a way that it guarantees a good spread of the contact points in the buffer.

After the replacement procedure, a search is performed to find the two points in the buffer that are farthest away from each other that still respect the spatial constant $d_{T}$. Then, the estimated normal $\vec{n}_{T}$ is defined as perpendicular to the line segment connecting those two points. Note that each time the slave breaks contact, the buffer is cleared out.

For the experiments, the trajectory based estimate $\vec{n}_{T}$ is considered reliable if the measure for the error is smaller than 0.25 , with this measure calculated as follows:

$$
\text { measure }=\frac{\sqrt{\left.\sum_{i=1}^{\mathrm{n}_{b}}\left(d_{i}\right)^{2}\right)}}{\text { Max. distance in buffer }}
$$

with $d_{i}$ the distance between the point $i$ and the estimated surface. In case the maximum distance between two points in the buffer is smaller than $75 \%$ of the spatial constant $d_{T}$, the estimate is always considered unreliable.

For the experiments, the buffer size $\mathrm{n}_{b}$ was set to 50. As mentioned above, for the PR2 arm, there is a big uncertainty of more than $10 \mathrm{~mm}$ on the position of the end-effector calculated based on the joint positions. Therefore, the spatial constant $d_{T}$ has to be set quite large, i.e. at $15 \mathrm{~mm}$. The drawbacks of a large spatial constant are the fact that the estimator is less sensitive for features with a small radius of curvature and the fact that a bigger amount of estimation lag is introduced in the loop.

\section{Camera information: $\vec{p}_{V}$ and $\vec{n}_{V}$}

Using the disparity image from the stereo-camera in the head of the PR2 robot, a local plane search is performed in front of the end-effector of the PR2-arm.

To select the search region, we need to forward-predict where the end-effector of the slave is likely to make contact with the environment. For simplicity, we assume that the endeffector is already close to the future contact point of the surface. Hence, the search region is defined as a rectangular region of 40x20 pixels around the projection of the end-effector into the image. In case the objects of interest are in the range of $0.8 \mathrm{~m}$ of the camera, which is the nominal distance the robot

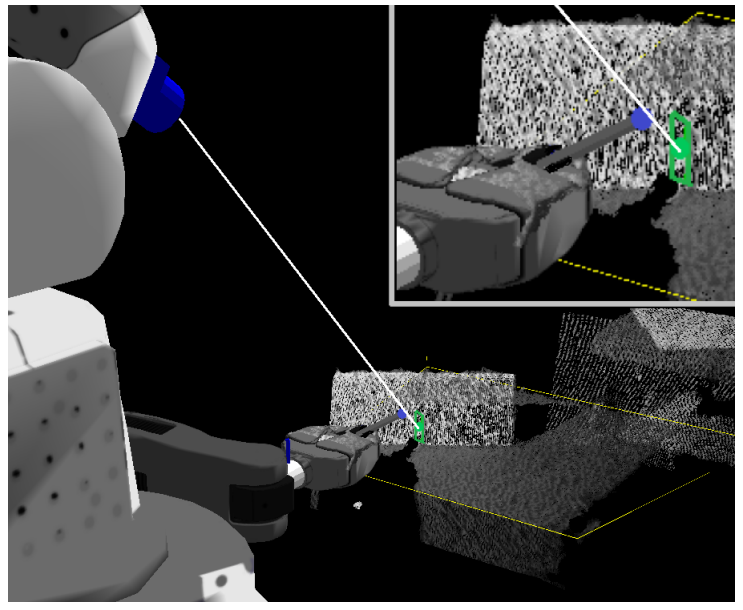

(a)

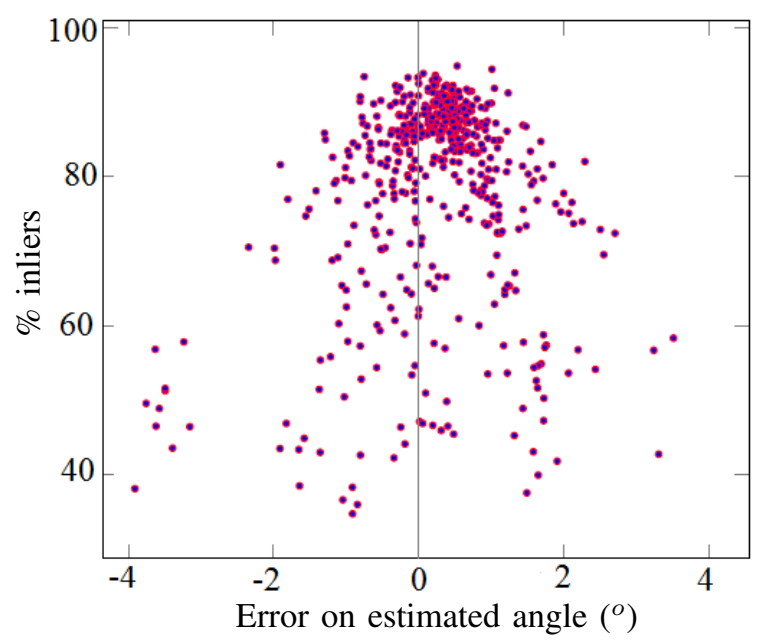

(b)

Fig. 2. The disparity image of the stereo-camera in the head of the PR2 robot is used to do a local plane search around the green dot. The detail in (a) shows this green dot that is a projection of the end-effector, marked by the blue dot, into the camera image. (b) The percentage of inliers with respect to the error on the estimated angle.

arm can reach, this comes down to a region of $30 \times 15 \mathrm{~mm}$. A topic of future work is to define a more general solution to select where to estimate the local plane, involving actual forward prediction of the slave movement.

Fig. 2(a) shows this scenario, with the estimated surface in green. The green dot in the center of that local plane corresponds to the point on the object surface that lies on the same projective ray as the end-effector, i.e. they project to the same pixel in the camera image. The algorithm performing the local plane search is based on RANSAC [19]. The output of the algorithm is an estimate for both the location $\vec{p}_{V}$ and the orientation $\vec{n}_{V}$ of the plane in front of the end-effector of the PR2-arm.

For several reasons, camera information can be unreliable:

- Especially when the end-effector is interacting with the environment, there can be occlusion.

- When the orientation of the object plane becomes parallel 
TABLE I

PERFORMANCE OF THE DISPARITY-BASED LOCAL PLANE SEARCH ALGORITHM

\begin{tabular}{|l||l|l|}
\hline Angle to camera axis & std(estimated angle) & std(estimated dist.) \\
\hline $0^{\circ}$ & $0.5^{\circ}$ & $1.9 \mathrm{~mm}$ \\
$34^{\circ}$ & $1.0^{\circ}$ & $4.1 \mathrm{~mm}$ \\
$54^{\circ}$ & $1.1^{\circ}$ & $6.5 \mathrm{~mm}$ \\
$75^{\circ}$ & $3.2^{\circ}$ & $16.6 \mathrm{~mm}$ \\
\hline
\end{tabular}

to the camera axis, the disparity image can become too sparse.

- Camera information is inherently affected by image noise.

Table II shows the standard deviation on the estimated angle and distance of the plane for four different orientations of the actual plane. For each orientation, the algorithm is applied to 500 images of the same static scenario. The distance from the camera to the plane varies from 0.6 to $0.8 \mathrm{~m}$. This table clearly shows that the measured data becomes more noisy, hence less reliable, when the orientation of the object plane becomes more parallel to the camera axis.

The above mentioned issues motivate why the modelestimation challenge cannot only be approached from a computer vision perspective. Clearly, when considering also compliant objects, the need for multi-modal estimation becomes even higher.

A good reliability measure for the result of the algorithm, is the percentage of inliers. Fig. 2(b) shows this percentage of inliers as a function of the error on the estimated orientation for 500 local plane estimations in case of a static scenario. This plot illustrates the general trend that if the error in the parameter estimate is large, then the corresponding percentage of inliers is low. For the experiments, the vision based estimate $\vec{p}_{V}$ and $\vec{n}_{V}$ are considered reliable if the percentage of inliers is higher than $65 \%$.

The PR2 controller updates the disparity image at a rate of $15 \mathrm{~Hz}$. Due to the presence of a considerable amount of noise on the estimate, some severe low-pass filtering is required which negatively influences the overall phase lag in the loop. The bandwidth of the low-pass filter is set at $1 \mathrm{~Hz}$ and thus the use of this information source has to be restricted to quasistatic or static situations.

\section{E. History: $\vec{p}_{H}$ and $\vec{n}_{H}$}

Information about the location and orientation of the surface at a previous contact point can provide an initial value for a new estimation. The reliability of this information depends on a spatial constant $d_{H}$ and a time constant $\tau_{H}$ : the further away from the previous contact point the less reliable this information becomes. The longer ago this previous contact point was found, the larger the chance that something has changed in the environment.

For the experiments, the history information is considered reliable if the current position of the slave is no more than $20 \mathrm{~mm}$ away from where the object was last touched. How much time passed since this last contact, is not taken into account.

\section{MODEL ESTIMATION}

A hierarchical approach has been defined to select what information source is used at every time step.

For the model parameter $\vec{p}$, i.e. the location of the model plane, the following rule applies: In contact, i.e. when $\left\|\vec{F}_{e}\right\|>$ $F_{\text {thres }}$, the position sensor based estimate $\vec{p}_{P}$ is considered most reliable and used. Else, if the slave is moving in free space and the camera information is reliable, the estimate from the local plane search algorithm is used, i.e. $\vec{p}_{V}$. In case the camera information is not reliable, the history information $\vec{p}_{H}$ is selected.

Similarly, for the model parameter $\vec{n}$, the following rule applies: In contact, the estimate based on the trajectory information, i.e. $\vec{n}_{T}$, is used if it is reliable. If not reliable, the vision based estimate $\vec{n}_{V}$ is used and this can be both when in contact or while moving in free space. If both the trajectory and the camera information are unreliable, the history information $\vec{n}_{H}$ is used. In case also the history information is unreliable, the model parameter $\vec{n}$ is re-initialized with the force sensor information, i.e. $\vec{n}_{F}$. Thereafter, by construction, the history information is reliable again.

\section{THE MODEL-MEDIATED CONTROLLER}

This section describes an implementation of a 2-d.o.f. model-mediated controller that uses the multi-sensor based model estimator described above. This controller consists of four components [6], [7]. At the slave side, the model estimation uses sensory data to continuously estimate the model for the environment. At the master side, this model is used by the model rendering to generate the haptic feedback. The human response to the haptic rendering is monitored by the task estimation to generate a task description. Back at the slave side, the task execution regulates the slave to accomplish the incoming task as well as possible using the current model of the environment.

\section{A. Model estimation}

The model estimator at the slave side, discussed in detail in the previous section, continuously updates the model parameters $\vec{p}_{s}$ and $\vec{n}_{s}$.

\section{B. Model rendering}

The model maintained at the master side is directly updated with the most recent incoming model parameters from the slave side:

$$
\vec{p}_{m}=\vec{p}_{s} \quad \text { and } \quad \vec{n}_{m}=\vec{n}_{s} .
$$

The virtual plane defined by these model parameters should behave as a one-sided pure stiffness in the direction of the normal $\vec{n}_{m}$. To realize this behaviour, a haptic proxy is used. The position of this proxy is the perpendicular projection of the actual position of the master onto the plane in case the master has virtually penetrated the plane. In front of the plane, the proxy tracks the master position. The force to be applied by the master is then:

$$
\vec{F}_{m}=K_{p}^{m}\left(\vec{x}_{\text {proxy }}-\vec{x}_{m}\right) .
$$


Because the environment is assumed to be rigid, i.e. infinitely stiff in the direction of the normal $\vec{n}$, the gain $K_{p}^{m}$ should be set as high as practically possible.

\section{Task estimation}

A simple task representation is employed to represent the user's intent, namely a location and an interaction force: $\vec{x}_{\text {task }}$ and $\vec{F}_{\text {task }}$. The force vector $\vec{F}_{\text {task }}$ is the interaction force between the human operator and the master, i.e. the force the operator is applying to the model and the position $\vec{x}_{\text {task }}$ is not the actual position of the master but the proxy location. As such, the task is feasible against the model, independent of any practical limitations on the gain $K_{p}^{m}$.

\section{Task execution}

In the $\mathrm{x} / \mathrm{y}$-plane, a hybrid controller is used in the same way as described in [17]: the slave is force controlled in the direction perpendicular to the model plane and position controlled in the direction tangent to the model plane:

$$
\vec{F}_{s}=\vec{n}_{s}\left\|\vec{F}_{\text {task }}\right\|+\left(1-\vec{n}_{s} \vec{n}_{s}^{T}\right)\left(\mathbf{K}_{p}^{s}\left(\vec{x}_{\text {task }}-\vec{x}_{s}\right)-\mathbf{K}_{v}^{s} \dot{\vec{x}}_{s}\right) .
$$

\section{EXPERIMENTAL RESULTS}

The experiments are subdivided in two parts. First, the behaviour of the model estimator itself is shown and in a second part, a bilateral experiment is performed. Both parts consider the scenario with the curved surface in the environment as shown in Fig. 1(a)

\section{A. Testing the model estimator}

To test the model estimator, data was recorded while the slave tracked the motion of the human operator in a unilateral teleoperation setting. The resulting slave trajectory is shown in Fig. 3(a), Apart from the first $950 \mathrm{~ms}$, the slave was continuously in contact during the recording of the data, i.e. the interaction force with the environment exceeded the threshold value $F_{\text {thres }}=1 \mathrm{~N}$.

For this data, Fig. 3(b) shows the estimated orientation based on the force, camera and trajectory information. This orientation is measured as the angle between the normal $\vec{n}$ and the $\mathrm{x}$-axis. Note that the estimates are only shown in case the reliability measure respects the threshold.

The effect of surface friction on the normal estimation based on the force vector is clear through the hysteresis-like behaviour. While moving to the left, the angle of the local plane with respect to the $\mathrm{x}$-axis is overestimated, while when moving to the right, this angle is underestimated.

The figure also shows that the estimates obtained from the camera and the trajectory information correspond quite well, with the trajectory-based estimates significantly smoother for a similar amount of phase lag. The downside of the trajectory-based information is that the resulting estimates also show some hysteresis-like behaviour. This behaviour originates from the fact that the points in the buffer typically lag the latest contact point while moving in one direction and the magnitude of this hysteresis behaviour thus depends on the spatial constant $d_{T}$. The camera-based information has the

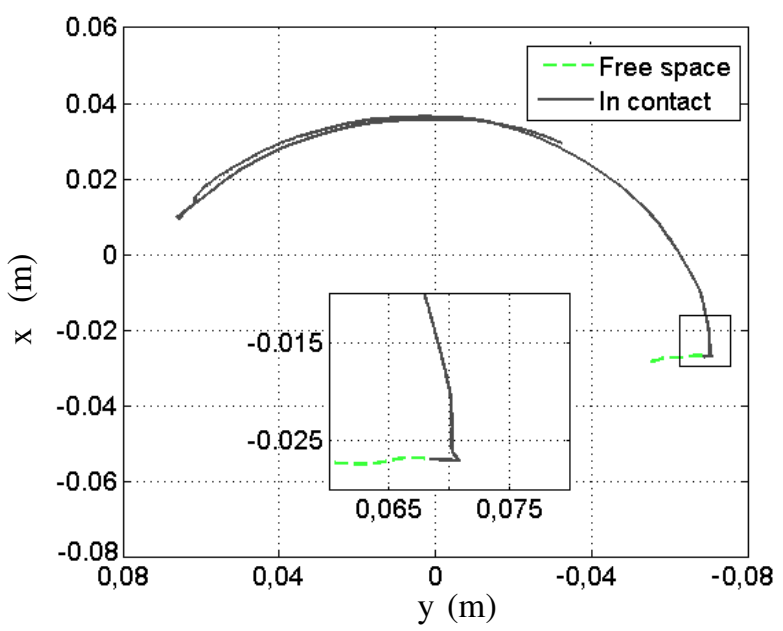

(a)

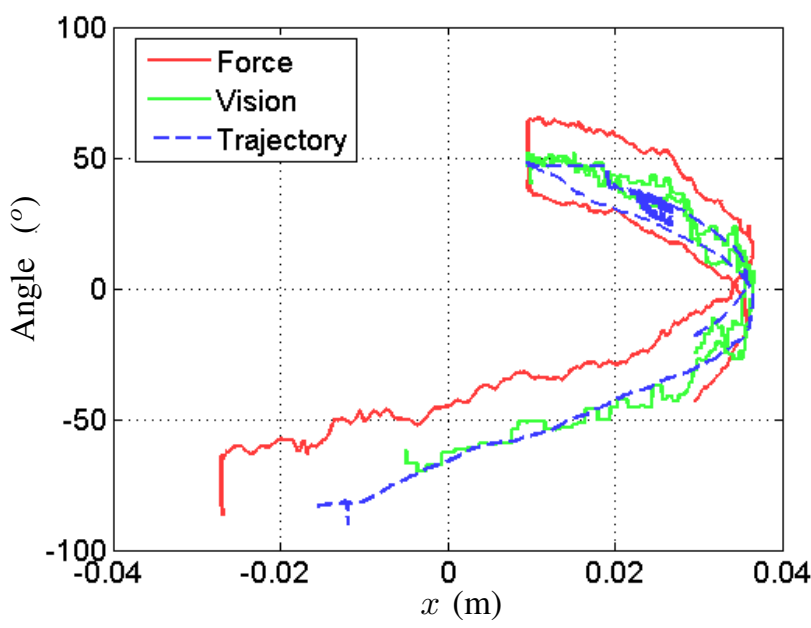

(b)

Fig. 3. (a) The slave trajectory that is followed during the recording of the sensor data used to test the model estimator. (b) The estimation of the normal based on three different information sources.

disadvantage that it is more noisy and subject to visibility constraints. If the end-effector is in contact with the environment it might occlude the contact point for the camera. The reliability of the model parameter estimates is also dependent on the relative alignment between surface normal and camera axis. This explains why there are no estimates shown for the vision sensor in Fig. 3(b) in case the angle is smaller than $-70^{\circ}$ : in that case the percentage of inliers is mostly smaller than 65 $\%$.

\section{B. Testing the bilateral controller}

During this second experiment a human operator interacted with the same curved environment shown in Fig. 1(a) The experiment shows distinct phases: first contact was made on the right side for a short period of time, then the operator moved towards the centre of the curved surface and finally slid to the left side and back.

Fig. 4 shows the data obtained during this experiment, i.e. the trajectory followed by the master and slave as well as the orientation of the interaction force between the master 

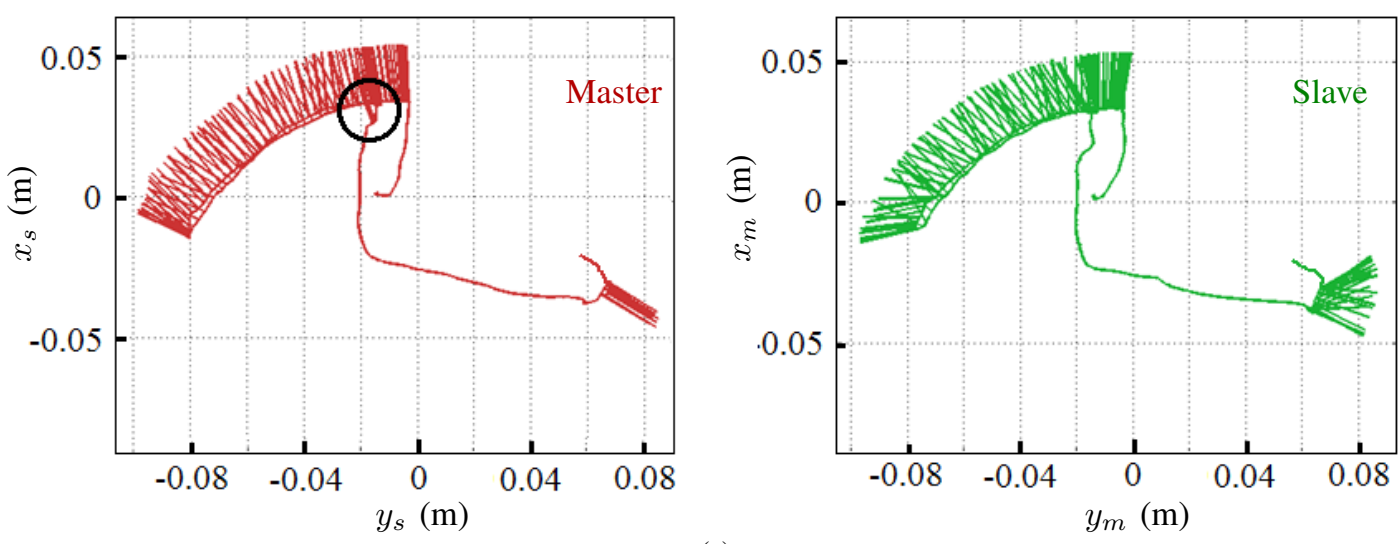

(a)

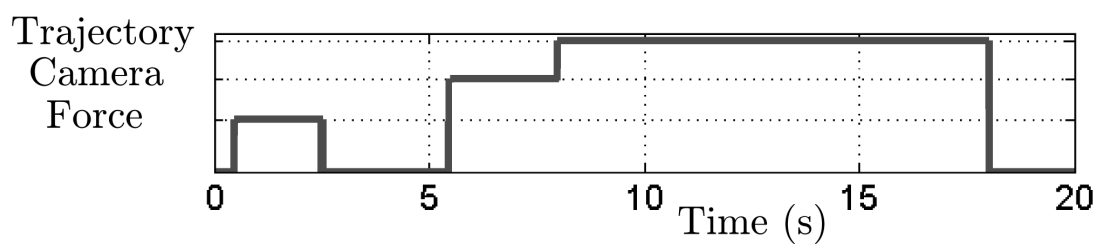

(b)

Fig. 4. A 2-d.o.f. model-mediated controller with sensor fusion: (a) the trajectory of the master and slave as well as the orientation of the interaction force. (b) The information source used to estimate the surface normal.

and the human operator and between the slave and the actual environment. This force orientation is only shown though, in case the interaction force exceeds the pre-defined threshold of $1 \mathrm{~N}$. Overall, one can see that the curved surface is reflected well to the human operator by the model-mediated controller.

Furthermore, Fig. 4(b) shows which source is used to estimate the surface normal at a certain moment in time:

Force When the environment is contacted at the right side, i.e. from 0.5 to 2.5 seconds, the initial estimate for the surface normal is based on the direction of the interaction force measured at the slave's end-effector upon initial contact. During the remainder of this period the trajectory information is not yet reliable since the range of motion is smaller than the spatial constant $d_{T}$. The camera information is not reliable either, since the camera-axis is almost perpendicular to the surface normal. Thus, as explained in section IV the history information is used, which continues to be the initial estimate.

Vision From 5.5 to 8 seconds, the slave moves towards and makes contact with the curved surface at a place where the camera is well aligned with the surface normal. During this period, the trajectory information is again unreliable and the camera information is the source used to estimate the surface normal. From 5.5 to 6.7 seconds the slave is not yet in contact, and the surface location is also estimated based on the camera information. Once the slave makes contact with the actual surface, i.e. after 6.7 seconds, the surface location is estimated based on the position measurement of the end-effector of the slave. Due to the compliance in the robotic arm, this means that the estimated surface location virtually penetrates the actual surface.

At the master side, this sequence of events entails that the human operator feels the object before the slave actually touches it. However, it also means that the operator feels the object moving slightly backwards. This signals the model adjustment due to the change in the information source from vision to position measurements. This phenomenon is visible in the small circle in Fig. 4.

Trajectory Once the range of motion around the initial contact point exceeds the spatial constant $d_{T}$, i.e. at 8 seconds, the trajectory information becomes reliable and can thus be used as the source for the estimation of the surface normal. The position measurement of the end-effector of the slave continues to be the source for the estimation of the surface location.

Note finally that this model-mediated controller reflects both the rigidity and the curvature of the environment to the human operator, while also providing a transparent feeling in free space. As long as the model estimator produces a reliable output, there are no stability problems despite the considerable amount of phase lag introduced by the limited performance of the slave.

\section{CONCLUSIONS}

This paper addressed the use of vision sensors for modelmediated teleoperation. Vision sensors are the common denominator of two important challenges for model-mediated teleoperation: (1) to robustly estimate geometric properties of the environment in a multi-d.o.f. scenario and (2) to predict environment properties before actually touching the environment.

For the specific case of estimating the orientation and location of a surface, this paper described several information sources including vision sensors with their respective reliability measures. A hierarchical approach has been defined to select what information source is used at every time step. 
The experiments showed the use of multiple information sources for robustly estimating model parameters during bilateral teleoperation. Also the ability to predict environment properties, before actually touching the environment, by using vision-based sensing is demonstrated.

Lastly, the bilateral experiment also demonstrates the fact, claimed in [6], [7], that the model for the environment should capture the locally most salient aspect of the environment, but can continuously adjust while the human operator explores the environment.

In future work, we would like to tighten the integration by performing actual sensor fusion. Instead of switching between modalities, the estimation should be based on all measurements and the time-dependent weighting factors at each time step. As such, the sensors can be used to continually cross-calibrate each other. Finally, we also want to explore dynamic environments, where information on compliance, friction and textures is obtained from touch-based sensing to augment the geometry obtained from vision-based sensing, i.e. to leverage even more the complementary nature of both sensing modalities.

\section{ACKNOWLEDGMENTS.}

This work was supported by a $\mathrm{PhD}$ grant from the Institute for the Promotion of Innovation through Science and Technology in Flanders (I.W.T.-Vlaanderen) and a travel grant from the Research Foundation - Flanders (F.W.O.-Vlaanderen)

\section{REFERENCES}

[1] G. Niemeyer and J.-J. Slotine, "Stable adaptive teleoperation," IEEE Journal of Oceanic Engineering, vol. 16, pp. 152-162, 1991.

[2] R. Daniel and P. McAree, "Fundamental limits of performance for force reflecting teleoperation," The Int. J. of Robotics Research, vol. 17, no. 8, pp. 811-830, 1998 .

[3] B. Hannaford, "A design framework for teleoperators with kinesthetic feedback," IEEE Transactions on Robotics and Automation, vol. 5, no. 4, pp. 426-434, August 1989.

[4] J. Funda and R. Paul, "Efficient control of a robotic system for timedelayed environments," in Fifth International Conference on Advanced Robotics (ICAR), Pisa, Italy, June 1991, pp. 219-224.

[5] P. Mitra and G. Niemeyer, "Model-mediated telemanipulation," The Int. J. of Robotics Research, vol. 27, no. 2, pp. 253-262, February 2008.

[6] J. Park, "Improving teleoperation by using models and tasks," Ph.D. dissertation, University of Stanford, 2009.

[7] B. Willaert, H. Van Brussel, and G. Niemeyer, "Stability of modelmediated teleoperation: Discussion and experiments," in Proceedings of the EuroHaptics Conference, Tampere, Finland, 2012, pp. 625-636.

[8] B. Willaert, E. Vander Poorten, D. Reynaerts, and H. Van Brussel, "A pragmatic method for stable stiffness reflection in telesurgery," in Proceedings of the EuroHaptics Conference, Madrid, Spain, June 2008, pp. 73-82.

[9] K. Hashtrudi-Zaad and S. Salcudean, "Adaptive transparent impedance reflecting teleoperation," in Proc. of the Int. Conf. on Robotics and Automation, Minneapolis, Minnesota, April 1996, pp. 1369-1374.

[10] S. Misra and A. Okamura, "Environment parameter estimation during bilateral telemanipulation," in Proc. of Symp. on Haptic Interfaces for Virtual Environment and Teleoperator Systems, Virginia, USA, March 2006, pp. 301-307.

[11] A. Achhammer, C. Weber, A. Peer, and M. Buss, "Improvement of model-mediated teleoperation using a new hybrid environment estimation technique," in Proc. of the Int. Conf. on Robotics and Automation, 2010, pp. 5358-5363.

[12] C.-P. Kuan and K.-Y. Young, "Challenges in vr-based robot teleoperation," in Proc. of the Int. Conf. on Robotics and Automation, 2003, pp. 4392-4397.
[13] G. De Gersem, H. Van Brussel, and J. Vander Sloten, "Enhanced haptic sensitivity for soft tissues using teleoperation with shaped impedance reflection," in Proceedings of the World Haptics Conference, Pisa, Italy, March 2005.

[14] H. Seraji and R. Colbaugh, "Force tracking in impedance control," The Int. J. of Robotics Research, vol. 16, no. 1, p. 97117, 1997.

[15] A. Fedele, A. Fioretti, C. Manes, and G. Ulivi, "On-line processing of position and force measures for contour identification and robot control," in Proc. of the Int. Conf. on Robotics and Automation, 1993, pp. 369374.

[16] D. Verscheure, J. Swevers, H. Bruyninckx, and J. Schutter, "On-line identification of contact dynamics in the presence of geometric uncertainties," in Proc. of the Int. Conf. on Robotics and Automation, 2008, pp. $851-856$.

[17] P. Mitra, "Model mediation for time-delayed teleoperation," Ph.D. dissertation, Stanford University, 2008.

[18] B. Willaert, B. Corteville, J. Van Vlem, K. Vanwynsberghe, D. Reynaerts, and H. Van Brussel, "A multi-purpose haptic d device for research on physical human-robot interaction," in Proceedings of the Int. Conf. on New Actuators (ACTUATOR), Bremen, 2012, pp. 530-533.

[19] "Fast plane detection algorithm. see http://www.ros.org/wiki/fast_plane_detection." see 